\title{
LA TRANSMISIÓN DE LOS ESTILOS DE GOBIERNO
}

Fernando Vizcaya Carrillo

Universidad Simón Bolívar. Venezuela

\begin{abstract}
"...El ideal democrático de educación será una ilusión y hasta una farsa trágica a menos que el ideal domine cada vez más nuestro sistema de educación pública"
\end{abstract}

John Dewey

Democracia y Educación, 110

\section{INTRODUCCIÓN}

El tema de este ensayo tiene como justificación, la posibilidad de profundizar en alguno de los aspectos enmarcados en la propuesta de investigación, la cual tiene como objeto la búsqueda de claridad en la problemática planteada - a veces de forma dramática-, de la continuidad en los sistemas de gobierno que tienden a la democracia.

El trabajo tiene como tema base el educar la ciudadanía para la democracia participativa. Una parte del trabajo será una aproximación al problema del gobierno de los hombres que incluye un aspecto educativo formal o la "Transmisión de los estilos de Gobierno". Allí enmarcamos este ensayo puesto que tiene que ver, por una parte con una implicación epistemológica del contexto investigativo y por otra parte, con la posibilidad de plantearse un problema de tipo instruccional, abarcando los aspectos del itinerario específico de la educación (enseñanza, aprendizaje, y proceso de transmisión).

Los apoyos de fuentes bibliográficas en el trabajo investigativo descansan en John Dewey, John Rawls y Alisdair McIntyre. El problema de la educación se explicita para estos autores en concepciones que van desde lo pragmático y lo liberal-individualista, hasta lo comunitario, en el sentido de participación plena en las decisiones y administración de problemas en cada uno de los segmentos de población. Esto, por supuesto y en todos los casos, estriba en última instancia en la verdadera transmisión de conocimientos y hábitos ciudadanos. "Para Dewey la educación es un proceso vital y habitual, tanto en términos individuales como sociales. Concepción que está íntimamente vinculada a su idea de la naturaleza humana individualizada e indica que el trabajo es virtud, expresión vital y habitual de esta naturaleza" (Geneyro, J.;1991:19).

Profundizar en este punto tiene un doble atractivo, el primero es el problema de la enseñanza en un contexto de vida y transmisión de estilo político de gobierno, el cual incluye el riesgo vital de que su fracaso llevaría a la no continuidad de un sistema de gobierno y por lo tanto de vida social; y por otra parte, el problema de "construcción" de un sistema político que permita la subsistencia de la naturaleza humana, que es social y que se debe basar por todo esto en estructuras habituales de comportamiento cívico.

Por una parte está la posición de Rawls que pretende separar la ética de la política como funcionalidad pragmáticamente viable y por otra parte está la posición de Maclntery de inevitabilidad ética en la acción humana y mucho más si esta es comunitaria. "La tentación de suprimir la inevitable tensión 
entre ética y política por uno de sus procedimientos (subsunción de la ética, o subsunción de la política o separación) será permanente, con resultados siempre peligrosos o perjudiciales el estado justo deviene inevitablemente en un totalitarismo aunque sea ilustrado; el realismo político no encontrará límites a la razón del Estado; y la disminución liberal entre lo privado y lo público conduce a la doble moral y a la esquizofrenia cívica" (Rubio Carracedo;1990:21). En todo caso la comparación entre ambos sistemas de pensamiento nos lleva a tener posibilidades apreciables y valiosas de investigación.

Es claro que ambos aspectos -liberal y comunitario- son parte de la tendencia a la educación en matices concretos - no sólo en conocimientos sino básicamente en hábitos- de los miembros de las distintas comunidades. Tomar este aspecto como eje central de la vida democrática constituye la búsqueda de acciones repetidas de relación que se fundamentan en la justicia, que significa una lucha o esfuerzo racional para resguardarnos de la barbarie que en algunas épocas han desolado a la especie humana. "Ahora bien, digamos que una sociedad está bien ordenada no sólo cuando está diseñada para promover el bien de sus miembros, sino cuando también está efectivamente regulada por una concepción de la justicia" (Rawls,J:1985:21).

Además de los objetivos buscados con este ensayo, quisiera profundizar en algunos aspectos epistemológicos que den solidez a los planteamientos posteriores del trabajo en sí. La posibilidad real de transmisión de unas disposiciones en los ciudadanos que comparten un "estilo" de gobierno, genera la motivación para el estudio más profundo de los niveles y parámetros del funcionamiento, o del no funcionamiento, de los sistemas actuales democráticos. Esto permitiría una base de aproximación a los diversos desatinos o distorsiones que se han sufrido y también de los diversos aciertos vividos y construidos en los últimos años. Problemas como "clientelismo político", "populismo"; "partidocracia"; "obsolencia de sistemas de elección", pero también la vida del sistema de participación a pesar de los obstáculos y su inclusión en las tradiciones del país, ha hecho imperativo que se puedan plantear nuevas búsquedas, las cuales, -en este escrito - tienen el sesgo de filosofía del hecho educativo, el cual pretende abarcar todo el entorno social en sus diversos aspectos. "Bien es verdad que las ciencias sociales que proceden en términos empírico-analíticos tienen también un concepto de totalidad; sus teorías son teorías de sistemas, y una teoría general tendría que referirse al sistema social en conjunto" (Habermas.J; 1996:22)

\section{EL PROBLEMA}

El concebir la educación como un proceso capaz de ser comprobado científicamente, tanto en el objeto como en el sujeto y en el proceso mismo, nos da un impulso de investigar con ánimo de ampliar un concepto "deweyano" que define a la democracia más como "un estilo o forma de vida con un método pedagógico" (Democracia y Educación), que estrictamente como un sistema de actividad específica política.

El tema tiene implicaciones epistemológicas puesto que toca de una manera concreta la necesidad de revisión profunda, en sus estructuras ontológicas, las disposiciones del hombre en cuanto ser social y por lo tanto necesitado de una acción prescriptiva, sino normativa, de relación con los demás. Esto en las primeras de cambio lo podríamos definir como la búsqueda y establecimiento —por comprobación sistemática- de ciertas acciones que conviene se repitan en el actuar diario de esa comunidad. Estos hábitos se convierten en costumbres de acciones económicas, educativas y de vida ordinaria, — de vida política - en general y que ellos sean capaces de transmitirse a otras generaciones. 
Es evidente que estas actividades humanas deben tener un fundamento suficientemente sólido en lo racional y ser suficientemente razonables para que subsistan, es decir, para ser no unas estructuras de superficie, comprobables sólo empíricamente, sino que resistan una visión crítica de estructura profunda, porque se radican en la naturaleza de la persona. "La idea directriz (de una teoría de la Justicia) es que los principios de la justicia para la estructura básica de la sociedad, son el acuerdo original. Son los principios que las personas libres y racionales interesadas en promover sus propios intereses aceptarían en una porción inicial de igualdad como definitorios de los términos fundamentales de su asociación" (Rawls, J; 1985:28).

En realidad estamos hablando de hábitos que constituirían la naturaleza de una sociedad que principia su vida de acuerdo con una base que podríamos llamar epistémica; donde los acuerdos sociales no están sujetos a unas circunstancias que circunscriben la acción y la determinan temporalmente, sino que "viven" en la naturaleza propia de cada persona de ese entorno. Se ha definido esta base social como justicia y sabemos que ésta tiene niveles que parecen estar de acuerdo al grado de civilización o racionalidad (entre otras cosas) de un ciudad o de un enclave social. Podríamos distinguir entre esos tipos de justicia: la simple retribución de la proporcionalidad de la equidad. "Considerada como una entidad efectiva del mundo real, la democracia ha sido concebida como un conjunto particular de instituciones y de prácticas políticas, un cierto cuerpo de doctrinas jurídicas, un orden económico y social, un sistema que asegura el logro de ciertos resultados deseables o un proceso singular para la adopción de decisiones colectivas obligatorias" (Dahl, R.;1991:14). La Justicia parecería ser definitivamente la base mas sólida de un sistema que pretende a la democracia como forma de vida. El problema que se plantea, llegados a este punto, es si es un hábito, o un conocimiento o algo que está en la naturaleza de las personas, es decir, algo que se posee de nacimiento. Cada una de estas posibilidades plantea un problema diferente en lo que a educación se refiere, entendida como formadora y transmisora de ese estilo de gobierno.

\section{LA ENSEÑANZA}

Uno de los grandes obstáculos para el sistema democrático, captado de esa manera por sus seguidores mas que por sus críticos, es el de las definiciones del objeto de esa posible enseñanza. No hay posibilidad real de transmisión si no se tiene suficientemente "captado" lo que se va a transmitir. Esto crea problemas, pues "enseñar democracia" no es enseñar a votar; o enseñar a discutir los temas que por su naturaleza son discutibles en una asamblea de ciudadanos. Es eso y mucho más que eso. Es -como decía Dewey - un modo de vivir y esto requiere comprometerse con ese estilo de vida. La transmisión, por lo tanto, se realiza de manera orgánica no simplemente de manera superficial y por explicitaciones del lenguaje. "Un término que significa muchas cosas no significa ninguna, y eso es lo que ha pasado con el término democracia, que hoy ya no es tanto una palabra con sentido limitado y específico, como la expresión de un vago apoyo a una idea popular" (Dahl, R.;1991:10)

$Y$ este punto, la enseñanza, requiere una especial atención en el campo de la investigación educativa pues es claro que si no hay enseñanza no hay transmisión y por lo tanto no hay permanencia de costumbres en el tiempo, ni de valores, ni de conocimientos, ni de criterios de verdad, es decir no puede haber continuidad de ese concebir las relaciones con los demás, de concebir ese modo de vivir.

Por esto, en primer lugar hay que definir el objeto de enseñanza, suponiendo el hecho de saber qué es la enseñanza. Con el ánimo de aclarar términos, podemos advertir que la enseñanza es mucho más que 
la simple disertación de un tema ante otras personas, no es solamente la clase magistral, ni la explicación de fenómenos en un laboratorio o en un campo de observación. La transmisión ocurre cuando existe una "comunicación de significados" reales entre dos elementos que se relacionan entre sí y que dichos elementos tienen una finalidad común también, la cual puede ser tanto para la acción como para la contemplación. "Zeus se compadeció entonces de la raza humana y encargó a Hermes que le proporcionara el pudor y la justicia. Pero debía hacerlo además, no según la división del trabajo como las demás artes, sino a todos y cada uno, ya que si solamente la tuvieran algunos, las ciudades no podrían subsistir" (Rubio Carracedo, J.;1990:19). Allí es donde interviene la labor del educador. Según Piaget, existe un "tercer estadio" de captación en el hombre, que es cuando se comienza a relacionar, a socializar, "es consciente del otro" y por lo tanto entra en una fase cooperativa saliendo de su fase inmanente, comienza allí la posibilidad de la justicia. "Infiere Piaget la existencia de tres tipos de reglas, cuyas relaciones será preciso determinar: la regla motriz, debida a la inteligencia motriz preverbal y relativamente independiente de toda relación social, la regla coercitiva debido al respeto unilateral, y la regla racional debido al respeto mutuo" (Rubio Carracedo, J;1996:22)

En esa última etapa se capta la necesidad, que está en la naturaleza del hombre, de la participación de otra persona para lograr fines, requiere la acción socializada y por lo tanto (aunque no espontáneamente) cooperativa. Hay que tener en cuenta que esta etapa requiere de un hábito específico que es la tolerancia, la cual en un estadio más avanzado la podríamos llamar solidaridad. Si en este momento se genera, producto de una enseñanza bien dirigida, una captación de valores personales y sociales, la esencia del juego democrático -entendido éste como la aceptación de reglas que hacen mas agradable la cooperación - se va produciendo la transmisión, que luego es "asegurada" por la normativa correspondiente al entorno contextual. "Piaget llega a la conclusión de que el sentido de la justicia, a pesar de poder ser reforzado naturalmente por las normas y el ejemplo de los adultos, es en gran parte independiente de estas influencias y no requiere para desarrollarse mas que la cooperación y la solidaridad entre los niños" (Rubio Carracedo, J.;1996:25)

No obstante todas las razones que podamos dar para proponer un sistema o una metodología, sigue existiendo un problema — que podríamos llamar gnoseológico- en la enseñanza y que mientras no se defina con precisión no podremos acertar en las soluciones. Por ejemplo, los programas oficiales de los ministerios públicos de enseñanza en los países democráticos están llenos de contenidos sobre la democracia y de actividades que en principio deberían llevar a la práctica democrática. Sin embargo, es fácil evidenciar que simplemente por recibir estos contenidos no se produce un alumno democrático. Quizás no hemos llegado a pasar de las estructuras superficiales de transmisión y por lo tanto no nos adentramos en contenidos semánticos del proceso, no estamos acertando en la verdad para la enseñanza, por eso me atrevo a llamar a esto un problema gnoseológico.

La ciudadanía - esencia de la democracia - requiere un proceso de aprendizaje (que muchas veces es independiente del de enseñanza) y éste (el aprendizaje de la ciudadanía) subsiste aparentemente mucho más en los hábitos que en los conocimientos. El término hábito lo podríamos definir como esas acciones conscientes y por lo tanto repetidas al considerarlas buenas para la naturaleza de la persona y para la comunidad en que las realiza. Es por ello interesante reflexionar sobre el hecho de que lo que constituye al ciudadano en su esencia social es la consideración del bien pero del bien común, es decir, el acuerdo social —consenso— sobre la finalidad de la acción cooperativa. "Por la naturaleza misma de las 
cosas, el hombre como parte de la sociedad, se ordena al bien común y a la obra común para la que se asocian los miembros de la sociedad, y renuncia, si es necesario a otras actividades por naturaleza mas nobles que las del cuerpo político, en aras de la comunidad" (Maritain,J.;1968:71).

El punto crítico se produce quizás cuando se plantea la necesidad de la permanencia del sistema democrático: los procesos de enseñanza de ese modo de vivir. Quizás sea redundante explicar que el típico modelo de enseñanza verdadera es el que se produce cuando el que enseña, hace lo que quiere transmitir, es decir, dice lo que hace.

De acuerdo con Mclntyre una práctica es: cualquier forma coherente y compleja de actividad humana cooperativa, establecida socialmente, mediante la cual se realizan los bienes inherentes a la misma mientras se intentan lograr los modelos de excelencia que le son apropiados a esa forma de actividad y la definen parcialmente, con el resultado de que la capacidad humana de lograr la excelencia y los conceptos humanos de los fines y bienes que conlleva, se extienden sistemáticamente (Naval, C;1995:113)

Este aspecto es de los más importantes de la teoría democrática como forma de vida. La permanencia en ella implica esos procesos a los cuales hemos estado tratando de aproximarnos. Quizás la dificultad más apremiante estriba en la mezcla o el paso entre las formas cognoscitivas de captación y las formas valorativas de juicio. Es decir, la relación entre el concepto y el valor o, que es lo mismo decir, la relación -vinculante-entre lo que se conoce y su valor moral, su valor ético. Es inevitable en el comportamiento humano esa relación. Se conoce e inmediatamente se hace un juicio valorativo de lo conocido y allí posiblemente existen las dificultades de producir una metodología suficientemente asertiva en relación a los valores éticos que se deben transmitir y plantea además el método de hacerlo como dificultad. "Todavía no hemos encontrado la fórmula para compaginar o, al menos, evitar tensiones entre ética y política, sin dañar irremediablemente a la una o a la otra, lo cual no significa que haya fracasado el planteamiento de la llustración, como piensa McIntyre (1981); es probable que nunca demos con tal fórmula justamente porque no existe, porque tales tensiones son inevitables e, incluso fuente de fecundidad para ambas" (Rubio Carracedo,J;1990:22)

\section{EL APRENDIZAJE}

Los procesos de captación, incorporación o de aprehensión de formas, estructuras, valores o criterios de valoración, se ubican generalmente en las estructuras profundas de la naturaleza humana y no sólo en la naturaleza racional o volitiva, abarca también los deseos y hábitos, todo el aspecto afectivo y del entorno. Parece que pertenece este proceso, en el caso de la democracia, al ser humano completo, con lo cual podríamos decir que es un proceso ontológico y no simplemente racional o conductual. Muchas de las teorías que intentan explicar el aprendizaje están fundamentadas en apreciaciones de tipo empírico deductivas, o en mediciones por comportamiento y por observación de conductas predecibles ante ciertas acciones. No obstante todo esto, el "medir" el comportamiento social es mucho más difícil que medir el comportamiento individual en el sentido de poder predecir la captación del consenso en los fines de las acciones cooperativas en los elementos que intervienen en esa actividad. "Por eso creo, como Habermas, que el problema fundamental de hoy es la conciliación del objetivo clásico de la política -permitir que los seres humanos lleven vidas buenas y justas en una comunidad política - con la demanda moderna del pensamiento social que es el conocimiento científico del funcionamiento de la sociedad" (Bernstein, J.;1989:22) 
La mezcla de inclinaciones, tendencias y deseos produce en la conducta humana consciente, algunas estructuras sociales que aseguran ese aprendizaje. El estado es una de esas instituciones que se crean por medio de la intersubjetividad del comportamiento humano. Y se constituye por la necesidad de permanencia de unas costumbres, que luego se especifican en leyes y se concretan en normas y reglamentos.

Sin embargo, uno de los grandes problemas es que esa estructura con mucha frecuencia se convierte en medio de opresión, medio de poder para conseguir fines egoístas personales o de grupos, en lugar de ser medio de servicio a los demás. Recordamos lo que decía Weber "Todas las formas políticas son organizaciones de fuerza" (Weber,M.;1987:668). Incluso si no tiene el ciudadano unas disposiciones al cambio - disenso - ancladas en la racionalidad, se convierte en un obstáculo real para la vida democrática. Impide el disenso ciudadano, actividad vital del contexto democrático. En muchos casos el consenso que también es parte vital del sistema, se convierte en obligación, con lo cual, el derecho al voto —por ejemplo-, al sufragio electoral, es una actividad que si no se realiza, se quisiera incluso penalizar, impidiendo la plena y más profunda conducta democrática, la del disentir o consentir en el ámbito ciudadano. "El consenso cívico, que es activo, significa que los ciudadanos «dan o rechazan su confianza con un completo conocimiento de causa» y, destaca Durkheim, el Estado nada puede hacer sin el consenso ciudadano" (Geneyro, J; 1991:95)

Esto plantea problemas complejos, pues la metodología del aprendizaje o para el aprendizaje, no es de fácil captación. No es "normativa" sino que se mueve más en el plano de la libertad de acción cívica, de las disposiciones interiores de convicciones personales y esto requiere un tipo de docente especial, un tipo de docente que sea capaz de tener la flexibilidad suficiente como para adaptarse a las situaciones más tirantes sin caer en situaciones de irrespetar la dignidad del hombre que actúa en ese sistema político.

El desconcierto que se puede generar en una estructura como la del trabajo ordinario de un ministerio público, por ejemplo, acostumbrado a normativas perennes y estáticas, rayando en el totalitarismo en muchos casos, hace que se mueva esa estructura en un plano de destrucción del Estado democrático más que de creación y recreación de ese estado. "En un gobierno autoritario la fuente de autoridad siempre es una fuerza externa y superior a su propio poder; de esta fuerza externa que trasciende el campo político, siempre derivan las autoridades su autoridad, es decir, su legitimidad y es con respecto a ellas que miden su poder" (Arendt, H:;1996:107)

La democracia necesita del ciudadano y este no nace sino que se forma, se capacita en un espacio, en un tiempo, aprendiendo lo necesario para convivir en un sitio - locus iurídico - con sus conciudadanos y respetando unas leyes a las cuales ha dado su parecer, sino para la aprobación por consenso, si por el estudio de ellas y con la posibilidad real -en una auténtica democracia participativa - de disenso de esas mismas leyes. Así, el educador en un sistema democrático no es el maestro de aula (solamente), es el legislador y el juez, es el padre de familia y el gendarme que cuida la ciudad, de mantener las leyes de consenso y de respetar la posibilidad de disenso. "La idea directriz (de una teoría de la justicia) es que los principios de la justicia para la estructura básica de la sociedad, son el objeto del acuerdo original. Son los principios que las personas libres y racionales interesadas en promover sus propios intereses aceptarían en una posición inicial de igualdad como definitorios de los términos fundamentales de su asociación" (Rawls, J.;1985:28). El método a seguir no es simplemente una serie de técnicas pedagógicas, apoyadas en recursos didácticos que sean novedosos o eficaces para la instrucción. El método pedagógico en una 
democracia participativa requiere de una coherencia de vida de las personas que deben transmitir ese estilo de gobierno, requiere de coherencia, de virtud ciudadana. "..será preciso insistir en que una educación moral completa exige no solamente la madurez del juicio moral, sino también la formación de hábitos de acción o virtudes morales, que conlleven a estrategias educativas específicas para potenciar la capacidad de autorregulación y el refuerzo de la voluntad racional comunicativa frente a los impulsos egoístas instintivos y el peso de los hábitos deficientes adquiridos" (Rubio Carracedo, J.;1996:67)

\section{A MODO DE CONCLUSIÓN}

Plantearse un sistema educativo que se convierta verdaderamente en raíz y centro de una forma de gobierno, y que ese sistema tenga unas características de cambio permanente, parecería una especie de callejón sin salida, de propósitos encontrados y de búsqueda de problemas que no tienen solución.

Sin embargo, es precisamente la posibilidad real de cambio —no sólo momentáneo, o coyuntural, sino estructural- lo esencial en un sistema educativo para la democracia. Crear un sano criticismo en los ciudadanos y crear ciudadanos son las piedras angulares de ese sistema. Lo contrario, un sistema que profundice y perpetúe un estilo de gobierno es propio de regímenes de corte totalitario y por lo tanto causa de una gradual deshumanización.

Y este planteamiento de entrada tiene que ver con una concepción del hombre y de su relación con los semejantes, con los seres -iguales que él- con los cuales convive. Necesita que también, esa antropología satisfaga las dimensiones de las capacidades corporales y de los deseos espirituales del hombre y una sociología que comprenda -en el sentido de espacio humano- todos los matices de convivencia comunitaria, de relación jurídica y una perspectiva comunicacional humana.

Las estructuras sociales en una democracia suelen tender hacia formas definidas de prácticas políticas y no al revés. Eso ha sucedido en diversos sistemas y maneras de gobierno que nos trae la historia. Lo mas conocido en ese sentido, es la división que se ha convertido en una polarización, de las tendencias liberales y de las tendencias comunitarias. "Tanto el liberalismo como el conservadurismo nacieron en un clima en el que la opinión pública oscilaba con violencia y están unidos el uno al otro, no sólo porque cada uno podría perder su sustancia misma sin la presencia de su oponente en el campo de la teoría y de la ideología, sino porque ambos enfoques se ocupan en primer lugar de devolver su puesto tradicional ya sea a la libertad, a la autoridad o a la relación entre ambas" (Arendt, H. (1996: 111).

Ambas pretenden explicar los diferentes movimientos sociales y eventualmente algunas tendencias. Sin embargo, estas teorías no explican suficientemente la posibilidad de transmisión de ese estilo de vida. $Y$ es claro que se necesita una teoría suficientemente definitoria, en el sentido de limitar los parámetros de acción del investigador y también del estadista que se debe enfrentar diariamente con problemas en la administración de las políticas regionales o las grandes políticas nacionales e incluso internacionales. "Las luchas simbólicas a propósito de la percepción del mundo social puede tomar dos formas diferentes. En el aspecto objetivo, se puede actuar por acciones de representaciones individuales o colectivas, destinadas a hacer ver y a hacer valer ciertas realidades (...) Por el lado subjetivo, se puede actuar tratando de cambiar las categorías de percepción y de apreciación del mundo social, las estructuras cognitivas y evaluativas; las categorías de percepción, los sistemas de clasificación, es decir, lo esencial, las palabras, los nombres que constituyen la realidad social tanto como la expresan..." (Bordieu, P. ;1987:137) 
Proponer una metodología concreta de enseñanza de la democracia es caer precisamente en el problema que tenemos presente en nuestros países. Decir que el aprendizaje se realiza de una forma o de otra en la persona humana es trasladar el problema a otro nivel sin resolverlo totalmente. Plantearse una forma de vida y un método pedagógico, es quizás la forma mas asertiva para la democracia. Un modo de observar, un modo de enfocar los problemas reflexionando y un modo de comunicar es lo que quizás en el fondo se debe buscar. Estos modos deberían generar estilos curriculares (que tengan en cuenta la persona humana en su dignidad), definir programas (sobre todo los de ciencias puras y los que generen hábitos de resolución de problemas) y producir actividades (haciendo énfasis en las ciencias sociales) que enriquezcan la educación formal, de manera que se vaya produciendo ciudadanía como un producto final o un perfil. Hacerlo explícitamente o con detalle en este escrito no parece pertinente, pero sí producir la motivación conceptual.

Se planteará así un gobierno democrático, que en principio requiere una igualdad teórica de las oportunidades del ciudadano; posibilidad de capacitarse en función de las libertades respectivas que potencialmente enriquecen los matices o faces de la personalidad humana; las disposiciones jurídicas que hacen segura la interacción social o por lo menos encauzarlas por senderos racionales y razonables. Todo esto producto de la deliberación serena y buscadora de bienes - fines - comunes y que se pueda producir en un proceso de transmisión tal, que genere realmente unas tendencias democráticas, de deliberación consenso y disenso - en las personas que viven en un espacio establecido y comparten un tiempo. "La crítica debe llegar a ser herramienta pedagógica vital, no solo porque irrumpe en las mistificaciones y distorsiones que silenciosamente trabajan detrás de las etiquetas y rutinas de las prácticas escolares, sino también porque modela una forma de resistencia y de pedagogía de oposición". (Giroux, Henry; 1992:91)

Los diversos matices que recubren nuestros problemas, los del país como de cualquiera de Latinoamérica, son quizá la forma en que captamos los gobiernos, esquivando responsabilidades, sin el hábito de solidaridad arraigado en nuestra estructura racional y de esta manera es imposible la consecución de unas formas democráticas reales. "No hay crecimiento democrático fuera de la tolerancia que a la vez que significa sustantivamente la convivencia entre desemejantes, no les niega, sin embargo el derecho a luchar por sus sueños" (Freire, P.;1996:20). La democracia no se hereda, se consigue siempre, se recrea en las formas y los estilos teniendo como base la dignidad de la persona y el respeto del otro como vehículo del diálogo.

\section{BIBLIOGRAFÍA}

ARendt, Hannah (1996): Entre el Pasado y el Futuro. Editorial Península. Barcelona. España

ARISTÓTELES (1974): Etica a Nicómaco. Revista de Occidente. Madrid.

Bernstein, J.R. (1989): La Reestructuración de la Teoría Social y Política. Fondo de Cultura Económica. México.

Bordieu, PierRe (1987): Cosas Dichas. Gedisa. París.

CAstells y De IPPOla (1981): Metodología y Epistemología de las Ciencias Sociales. Editorial Ayuso. Madrid

DAHL, RoBeRT (1990): La Democracia y sus Críticos 
Tecnos Editorial. Madrid

DEWEY, JOHN(1978): Democracia y Educación, Editorial Losada. Buenos Aires.

FREIRE, PAULO (1996): Política y Educación. Editorial Siglo XXI. Madrid

GeneYro, JuAn CaRlos (1991): La Democracia Inquieta. (Durkheim y Dewey). Antrophos. Barcelona

GIDDENS, ANTONHY y OTROS (1990): La teoría Social hoy, Alianza Universidad. Madrid.

Habermas, Jürgen (1996): La Lógica de las Ciencias Sociales. Editorial Tecnos. Madrid

MACINTYRE, ALASDAIR (1987): Tras la Virtud, Editorial Crítica. Barcelona.

MARITAIN, JAQUes (1968): La Persona y el Bien Común. Club de Lectores. Buenos Aires 1968

NAVAL, CONCEPCIÓN (1995): Educar Ciudadanos. EUNSA. Pamplona.

PIEPER, JOSEF (1974): El Ocio y la Vida Intelectual. Editorial Rialp. Madrid

RAWLS, JoHN (1985): Teoría de la Justicia. Fondo de Cultura Económico. México

Rubio CARRACedo, José (1996): Educación Moral, Postmodernidad y Democracia. Editorial Trotta. Madrid (1990): Paradigmas de la Política. Editorial Antrophos. Barcelona.

Weber, MAX (1987): Economía y Sociedad. Fondo de Cultura. México. 


\title{
Contactar
}

Revista lberoamericana de Educación

\author{
Principal OEI
}

